\title{
Drug-resistance - a challenge for tuberculosis control in the European Union and European Economic Area
}

M J van der Werf (Marieke.vanderWerf@ecdc.europa.eu) ${ }^{1}$, M Sprenger ${ }^{1}$

1. European Centre for Disease Prevention and Control (ECDC), Stockholm, Sweden

Citation style for this article:

van der Werf MJ, Sprenger M. Drug-resistance - a challenge for tuberculosis control in the European Union and European Economic Area . Euro Surveill.

2014;19(11):pii=20737. Available online: http://www.eurosurveillance.org/ViewArticle.aspx?Articleld=20737

Article submitted on 11 March 2014 / published on 20 March 2014

Worldwide resistance to antituberculosis drugs is jeopardising the control and eventually the elimination of tuberculosis (TB). Patients who are infected by Mycobacterium tuberculosis resistant to antituberculosis drugs, especially to both isoniazid and rifampicin and thus having multidrug-resistant (MDR) TB, need more complicated treatment that lasts longer and consists of a large number of drugs and frequently leads to side effects. In the European Union and European Economic Area (EU/EEA), treatment outcome results for all types of drug resistance were below the target of $85 \%$ set by the European Centre for Disease Prevention and Control (ECDC), for new pulmonary culture-positive cases and with $34 \%$ the reported treatment success rate of MDR-TB cases was substantially below the target of $70 \%[1,2]$. Of TB cases diagnosed with MDR-TB in 2009 in the EU/EEA, almost two-thirds died during treatment, failed their treatment or defaulted from treatment. This failure to ensure adequate MDR-TB treatment not only puts patients' lives at risk but also paves the way for XDR TB, and further transmission of the disease.

Analysis of the worldwide data for TB from 2007 to 2010, showed proportions of MDR-TB of $3.4 \%$ in new TB cases and $19.8 \%$ in those previously treated [3]. The proportions of TB cases with MDR-TB are similar in the EU/EEA with $2.6 \%$ of the new culture-confirmed TB cases diagnosed with MDR-TB and $18.8 \%$ of previously treated culture-confirmed TB cases [2]. In this issue of Eurosurveillance and in the 13 March issue, studies on surveillance of drug-resistant TB, including molecular surveillance, are published. These studies show that drug-resistant TB occurs in diverse settings in Europe and that there is ongoing transmission of drug-resistant TB also in countries with a low TB incidence.

Most studies on TB and drug resistance focus on multidrug resistance. Using data submitted to the EU/EEA TB surveillance system, ECDC performed an analysis of all types of drug-resistant TB notified in the EU/EEA in the period from 2007 to 2012 [4]. In this six-year period, the proportion of TB cases with different drug resistance patterns has been stable with about $90 \%$ of the new laboratory-confirmed TB cases pan-susceptible, $6 \%$ monodrug-resistant, $2 \%$ polydrug-resistant, $2 \%$ MDR-TB, excluding extensively drug-resistant TB (XDR-TB), and $0.2 \%$ XDR-TB.

The fact that drug resistance does not seem to increase in the EU/EEA is good news in principle. However, with proportions of MDR-TB ranging from 20.6 to $46.7 \%$ in laboratory confirmed cases in neighbouring countries [2], EU/EEA countries need to be vigilant and prepared for tackling an increase in drug resistance. This is supported by the results from the study by Jenkins et al. in this issue, who analysed routinely collected surveillance data in Georgia [5]. They identified between January 2009 and June 2011, 1,795 incident MDR-TB cases confirmed through DST leading to a nationwide MDR-TB incidence of 16.2 notified cases per 100,000 population with considerable geographic variation from 0.0 to 5.0 for new MDR-TB cases and from 0.0 to 18.9 for previously treated cases. In prisons incidence was as high as 837 per 100,000.

A study by $C$ Ruesen et al. from the Netherlands also evaluated all types of TB drug resistance [6]. In contrast to the overall findings for the EU/EEA, they revealed that antituberculosis drug resistance increased in the Netherlands since 1993 in patients born in the Netherlands, and since 2005 , in foreign-born patients. Furthermore, only a small fraction ( $8 \%$ ) of the identified cases seemed to have acquired drug resistance, while most (92\%) were considered to be infected by a resistant strain. Most transmission of drug resistant TB, over $60 \%$, occurred before 1993 or abroad. In only $9 \%$ of the cases the transmission definitely took place in the Netherlands.

A molecular surveillance study in Switzerland including all 49 MDR-TB strains identified in the period 2006 to 2012 , showed that 12 strains were grouped into six clusters [7]. In-country transmission was likely in four clusters. Most other strains were obtained from MDR-TB cases of foreign origin and were likely imported to Switzerland. 
Also in the current issue of Eurosurveillance, transmission of MDR-/ XDR-TB strains across the borders of EU/EEA countries is presented based on data from a ECDC initiated molecular surveillance project on MDR-/XDR-TB in the EU [8]. Cross-border transmission of MDR-TB was defined as at least two strains with identical 24-locus variable number of tandem repeat (VNTR) typing patterns, were the cases are in at least two different EU/EEA countries. Almost half ( $45 \%$ ) of the strains collected proved to be part of international European clusters and of these, $60 \%$ were part of a single, large European cluster. Real transmission of MDR-/XDR-TB, however, is likely to be higher since the project only covered $12 \%$ of the total number of MDR-/ XDR-TB cases notified in the in the period 2003-2011.

The above studies show that MDR-TB is both imported into and transmitted within the EU/EEA. To prevent transmission of MDR-TB early diagnosis, including use of rapid tests for diagnosis of drug resistance, infection control, and contact investigation are essential. Furthermore, transmission of TB is prevented by timely and adequate treatment which shortens the infectious period. According to a recent survey in Europe, tracing of contacts has been implemented in all participating EU/EEA countries. While this is very positive, the survey also showed that rapid diagnostic tests are not available in all countries, nor is TB infection control implemented everywhere [9]. This demonstrates that there is room for more rigorous implementation of measures to avoid transmission of TB and MDR-TB in the EU/EEA.

To prevent importation of TB and MDR-TB, migrant TB screening seems to be an attractive intervention. Eleven EU countries have implemented migrant screening [10]. All respective screening programmes reported the use of chest radiography to screen for active disease. The yield of this screening is however, low $[10,11]$. Also, use of chest radiography screening will not identify individuals infected with TB but not yet symptomatic. Therefore, it is of utmost importance that healthcare systems are accessible for migrants and that healthcare is affordable for them so that they can search for care as soon as symptoms of active TB develop.

MDR-TB can only be diagnosed when $M$. tuberculosis bacilli are present in sputum or other samples. Since TB in children is often paucibacillar, laboratory confirmation can be difficult [12]. Of all paediatric cases reported to the EU/EEA TB surveillance system between 2000 and 2009 , only $16.9 \%$ were confirmed by culture [13]. Sanchini et al. in this issue, [14] analysed routine laboratory data of several national TB reference laboratories in the EU to assess laboratory procedures for diagnosis of paediatric TB. All laboratories receiving primary samples performed the whole range of diagnostic tests, i.e. smear microscopy, molecular identification, culture, and first- and second-line drug susceptibility testing. In the period 2007-2011, 156 of $5,156(3.0 \%)$ samples from children tested positive for
Mycobacterium tuberculosis (complex) and of these $10(6.4 \%)$ showed multidrug resistance. This is higher than the $4.7 \%$ MDR TB reported for all TB cases in 2012 [4]. Thus MDR-TB cannot be neglected in children and intensive efforts should be applied to collect samples from children for laboratory confirmation of TB. Since children are often not able to produce sputum samples spontaneously, gastric aspiration and sputum induction may be applied instead [15]. Also, even though more invasive, fine-needle aspiration biopsy has proved to be useful for collecting samples in children with a peripheral lymph-node mass [16]. In addition to facilitating the diagnosis of TB and drug resistance, strains obtained from positive cultures can also be subjected to molecular typing and provide valuable information about transmission of TB such as demonstrated in several studies in this issue $[6,7,8]$.

Since unsuccessful treatment outcomes are reported frequently within the EU/EEA, especially for MDR-TB cases, those involved in treatment and care of MDR-TB patients should do everything possible to arrive at better treatment outcomes [2]. To assist healthcare workers with the management of drug-resistant TB cases several tools were developed. First of all, the 'European Union Standards for Tuberculosis Care', these include standards for treatment of TBs, including MDR-TB $[17,18]$. In addition, a consensus statement on management of patients with M/XDR-TB in Europe has been developed recently [19]. A specific tool to assist healthcare workers is the 'Electronic Consilium' which was launched to provide scientifically sound and evidence-based clinical consultation for drug-resistant TB and other difficult-to-treat TB cases [20]. Between the start of the consilium in September 2012 and July 2013, the platform has supported the clinical management of ten TB patients [21]. We hereby call on all healthcare workers to make use of the consilium and other tools to guarantee proper management of patients with drug resistant TB and to cure them

In conclusion, early diagnosis of all cases with MDR-TB, adequate treatment of MDR-TB patients and implementation of infection control measures to prevent further transmission, are crucial to overcome challenges posed by MDR-TB. Realising the full implementation of these prevention and control measures should be a priority for policy makers and healthcare workers engaged in controlling and eliminating TB and MDR-TB.

References

1. European Centre for Disease Prevention and Control (ECDC). Progressing towards TB elimination. A follow-up to the Framework Action Plan to Fight Tuberculosis in the European Union. Stockholm: ECDC; 2010. Available from: http:// ecdc.europa.eu/en/publications/publications/101111_spr_ progressing_towards_tb_elimination.pdf

2. European Centre for Disease Prevention and Control (ECDC)// WHO Regional Office for Europe. Tuberculosis surveillance and monitoring in Europe, 2014. Stockholm: ECDC / WHO Regional Office for Europe; 2014. Available from: http://www.euro. who.int/_data/assets/pdf_file/o004/245326/Tuberculosissurveillance-and-monitoring-in-Europe-2014.pdf 
3. Zignol M, van Gemert W, Falzon D, Sismanidis C, Glaziou P, Floyd K, et al. Surveillance of anti-tuberculosis drug resistance in the world: an updated analysis, 2007-2010. Bull World Health Organ. 2012;90(2):111-9D. http://dx.doi.org/10.2471/ BLT.11.092585

4. van der Werf MJ, Ködmön C, Hollo V, Sandgren A, Zucs P. Drug resistance among tuberculosis cases in the European Union and European Economic Area, 2007 to 2012. Euro Surveill. 2014;19(10): pii=20733. Available from: http://www. eurosurveillance.org/ViewArticle.aspx?Articleld=20733

5. Jenkins HE, Gegia M, Furin J, Kalandadze I, Nanava U, Chakhaia T, Cohen T. Geographical heterogeneity of multidrug-resistant tuberculosis in Georgia, January 2009 to June 2011. Euro Surveill. 2014;19(11): $\mathrm{pii}=20743$.

6. Ruesen C, van Gageldonk-Lafeber AB, de Vries G, Erkens CG, van Rest J, Korthals Altes $H$, de Neeling $H$, Kamst $M$, van Soolingen D. Extent and origin of resistance to antituberculosis drugs in the Netherlands, 1993 to 2011. Euro Surveill. 2014;19(11): $\mathrm{pii}=20738$.

7. Somoskovi A, Helbling P, Deggim V, Hömke R, Ritter C, Böttger EC. Transmission of multidrug-resistant tuberculosis in a lowincidence setting, Switzerland, 2006 to 2012 . Euro Surveill. 2014;19(11): pii=20736.

8. De Beer JL, Ködmön C, van der Werf MJ, van Ingen J, van Soolingen D, the ECDC MDR-TB molecular surveillance project participants. Molecular surveillance of multi- and extensively drug-resistant tuberculosis transmission in the European Union from 2003 to 2011. Euro Surveill. 2014;19(11): pii=20742.

9. D’Ambrosio L, Dara M, Tadolini M, Centis R, Sotgiu G, van der Werf MJ, et al. Tuberculosis elimination: theory and practice in Europe. Eur Respir J. 2014. [Epub ahead of print]. http://dx.doi. org/10.1183/09031936.00198813

10. Klinkenberg E, Manissero D, Semenza JC, Verver S. Migrant tuberculosis screening in the EU/EEA: yield, coverage and limitations. Eur Respir J. 2009;34(5):1180-9. http://dx.doi. org/10.1183/09031936.00038009

11. Severi E, Maguire H, Ihekweazu C, Bickler G, Abubakar I, editors. Migrant tuberculosis screening: very low yield of active tuberculosis at port of entry and prediction for diagnosis after entrance - United Kingdom, 2009/2010. 2012; European Scientific Conference on Applied Infectious Disease Epidemiology (ESCAIDE): Edinburgh. Available from: http:// ecdc.europa.eu/en/ESCAIDE/Materials/Presentations $\% 20$ 2012/ESCAIDE2012_Session_3_2_Fintry.pdf

12. Zar HJ, Connell TG, Nicol M. Diagnosis of pulmonary tuberculosis in children: new advances. Expert Rev Anti Inf Ther. 2010;8(3):277-88. http://dx.doi.org/10.1586/eri.10.9

13. Sandgren A, Hollo V, Quinten C, Manissero D. Childhood tuberculosis in the European Union/European Economic Area, 2000 to 2009. Euro Surveill. 2011;16(12):pii=19825. Available from: http://www.eurosurveillance.org/Public/Articles/ Archives.aspx

14. Sanchini A, Fiebig L, Drobniewski F, Haas W, Richter E, Katalinic-Jankovic $\mathrm{V}$. Laboratory diagnosis of paediatric tuberculosis in the European Union/European Economic Area: analysis of routine laboratory data, 2007 to 2011. Euro Surveill. 2014;19(11): $\mathrm{pii}=20744$.

15. World Health Organization (WHO). Guidance for national tuberculosis programmes on the management of tuberculosis in children. 2006: WHO; Geneva.

16. Wright CA, Warren RM, Marais BJ. Fine needle aspiration biopsy: an undervalued diagnostic modality in paediatric mycobacterial disease. Int J Tuberc Lung Dis. 2009;13(12):1467-75.

17. Migliori GB, Zellweger JP, Abubakar I, Ibraim E, Caminero JA, De Vries G, et al. European union standards for tuberculosis care. Eur Respir J. 2012;39(4):807-19. http://dx.doi. org/10.1183/09031936.00203811

18. van der Werf MJ, Sandgren A, D’Ambrosio L, Blasi F, Migliori GB. The European Union standards for tuberculosis care: do they need an update? Eur Respir J. 2014. Forthcoming.

19. Lange C, Abubakar I, Alffenaar JWC, Bothamley G, Caminero JA, Carvalho ACC, et al. Management of patients with M/XDR TB in Europe - a TBNET consensus statement. Eur Respir J. 2014. Forthcoming.

20. Blasi F, Dara M, van der Werf MJ, Migliori GB. Supporting TB clinicians managing difficult cases: the ERS/WHO Consilium. Eur Respir J. 2013;41(3):491-4. http://dx.doi. org/10.1183/09031936.00196712

21. D’Ambrosio L, Tadolini M, Dupasquier S, Tiberi S, Centis R, Dara M, et al. ERS/WHO Tuberculosis Consilium: reporting of the initial 10 cases. Eur Respir J. 2014;43(1):286-9. http:// dx.doi.org/10.1183/09031936.00125813 